Leomar VIANA ${ }^{1}$

Roberto Calderon

GONÇALVES ${ }^{1}$

José Paes de OLIVEIRA

$\mathrm{FILHO}^{1,2}$

Antônio Carlos PAES

Simone Biagio $\mathrm{CHIACCHIO}^{1}$

Marcio Garcia RIBEIRO ${ }^{1}$

Correspondência para:

José Paes de Oliveira Filho-Departamento de Clínica Veterinária da FMVZ - UNESP Botucatu, Distrito de Rubião Jr. S/N CEP 18610-000- Botucatu - São Paulo. FONE /FAX1438116067,ze.filho@uol.com.br

Recebido para publicação: 24/07/2006 Aprovado para publicação: 25/06/2007

\title{
Susceptibilidade in vitro a antimicrobianos da Mannheimia haemolytica e da Pasteurella multocida isoladas de ovinos sadios e com doenças respiratórias
}

\author{
1 - Faculdade de Medicina Veterinária e Zootecnia da Universidade Estadual \\ Paulista, Botucatu-SP \\ 2 - Faculdade de Medicina Veterinária e Zootecnia de Garça - ACEG, Garça-SP
}

\section{Resumo}

Pasteurella multocida e Mannheimia haemolytica (P. haemolytica) estão associadas a enfermidades no sistema respiratório de ovinos. Com o objetivo de avaliar a susceptibilidade in vitro destes microrganismos frente aos antimicrobianos, foram colhidas amostras de nasofaringe $(n=180)$ e orofaringe $(n=82)$ de ovinos com e sem enfermidade respiratória. Dentre os antimicrobianos testados, a sensibilidade foi maior para enrofloxacina (100\%) e florfenicol (100\%), considerandose ambas as espécies bacterianas. Observou-se resistência de $M$. haemolytica e $P$. multocida à tetraciclina $(15,64 \%$ e $17,65 \%$, respectivamente) e penicilina ( $1.82 \%$ e $4.2 \%$, respectivamente).

\section{Introdução}

Mannheimia haemolytica é o agente etiológico mais comumente isolado de ovinos com doenças respiratórias nos países de clima temperado. Por sua vez, em países de clima tropical a Pasteurella multocida, que era considerada incomum em ovinos, vem se destacando como um dos principais causadores desta modalidade de doença. ${ }^{1}$ A pasteurelose é a enfermidade respiratória que mais danos traz à indústria ovina. ${ }^{2} \mathrm{O}$ conhecimento da etiologia das afecções respiratórias é importante tanto para a instituição de uma boa terapia como para a implantação de medidas profiláticas que, na maioria das criações de ovinos, limitar-seiam a simples modificações de manejo. ${ }^{3}$

Segundo Diker, Akan e Harizoglu, são poucos os antimicrobianos que se encontram disponíveis para o uso específico em pequenos ruminantes, sendo a penicilina $G$ procaína, a tetraciclina e a sulfa associada ao trimetoprim, os mais utilizados. O florfenicol é um antibiótico relativamente novo que ainda não está comercialmente disponível para o uso em pequenos ruminantes, mas que apresenta uma alta efetividade no tratamento da pasteurelose pneumônica e septicêmica dos ovinos e caprinos ${ }^{4}$, assim como a enrofloxacina. ${ }^{5}$

Considerando os prejuízos causados pelas doenças respiratórias em ovinos e a limitação de informações sobre a doença no Brasil, o presente estudo procurou investigar a susceptibilidade in vitro aos antimicrobianos da Pasteurella multocida e Mannheimia haemolytica isoladas de nasofaringe e de orofaringe de ovinos clinicamente sadios ou portadores de alterações do sistema respiratório.

\section{Material e Método}

Foram colhidas amostras de nasofaringe e de orofaringe de 180 ovinos clinicamente sadios e de 82 ovinos com enfermidades respiratórias, provenientes de 17 fazendas da região de Botucatu - SP, amostrados aleatoriamente independentemente de sexo, idade e raça. $\mathrm{O}$ exame físico, realizado segundo os critérios clínicos adotados por Stöber ${ }^{6}$, determinou os animais portadores de alterações 
respiratórias.

Antes da colheita do material proveniente da nasofaringe de cada animal, realizou-se a anti-sepsia da área externa das narinas, com algodão embebido em álcool iodado a $5 \%$. Os swabs nasofaringeanos foram guiados por sonda de polietileno de silicone estéril. Para a colheita das amostras de orofaringe com swab, utilizou-se abaixador de língua próprio, para visualizar e delimitar a área a ser amostrada (tonsilas). As amostras foram depositadas em tubos de ensaio contendo $5 \mathrm{~mL}$ de meio de transporte (Stuart), acondicionadas imediatamente em caixas isotérmicas sob temperatura de refrigeração e encaminhadas ao Serviço de Diagnóstico Microbiológico de Enfermidades Infecciosas dos Animais, da Faculdade de Medicina Veterinária e Zootecnia da UNESP de Botucatu-SP, onde foram processadas.

Após isolamento, identificação e classificação segundo a metodologia descrita por Viana 7, 275 cepas de $M$. haemolytica e 119 cepas de $P$. multocida foram submetidas a testes in vitro de susceptibilidade a antimicrobianos, utilizando-se o método de difusão ${ }^{8}$, em ágar Müeller-Hinton enriquecido com $5 \%$ de sangue desfibrinado de ovino. Foram testados os seguintes antimicrobianos: tetraciclina (TET - 30mcg), enrofloxacina (ENO - 5mcg), sulfametoxazol + trimetoprim (SUT - 25mcg), penicilina (PEN - $10 \mathrm{UI}$ ), ciprofloxacina (CIP - 5mcg) e florfenicol (FLF - 30mcg).

\section{Resultados}

Os maiores índices de sensibilidade, tanto para $M$. haemolytica quanto para $P$. multocida, foram observadas frente ao florfenicol $(100 \%)$ e enrofloxacina $(100 \%)$. De forma semelhante, o sulfametoxazol associado ao trimetoprim, a ciprofloxacina e a penicilina, foram eficazes tanto para $M$. haemolytica (99,64\%, 99,64\% e 96\%, respectivamente - Tabela 1) quanto para $P$. multocida $(99,16 \%, 98,32 \%$ e $94,12 \%$, respectivamente - Tabela 2). Em contraste, os maiores índices de resistência, tanto da
M. haemolytica (Tabela 1) quanto da $P$. multocida (Tabela 2) foram constatados frente à tetraciclina $(15,64 \%$ e $17,65 \%$, respectivamente).

\section{Discussão}

Dentre os antimicrobianos utilizados, verificou-se alta sensibilidade in vitro por parte das cepas testadas, frente à enrofloxacina, florfenicol e ciprofloxacina. Estes dados concordam com os obtidos em estudos conduzidos em outros países, que referem alta sensibilidade à enrofloxacina e ao florfenicol, de cepas de $M$. haemolytica e de P. multocida, isoladas de casos de pasteurelose pneumônica em ovinos. ${ }^{4,5}$ Entretanto, não foi possível encontrar na literatura consultada, dados referentes à efetividade in vitro da ciplofloxacina, frente às linhagens de $M$. haemolytica e de P. multocida isoladas do sistema respiratório de ovinos.

O sulfametoxazol associado ao trimetoprim mostrou boa efetividade in vitro, ao contrário do observado por Mackie, Barton e Hindmarsh ${ }^{5}$, que verificaram alta resistência a estes antimicrobianos por cepas de $M$. haemolytica isoladas de processos septicêmicos em ovinos.

Os maiores índices de resistência tanto nas amostras de $M$. haemolytica, quanto de $P$. multocida, ocorreram com a tetraciclina e menor escala, com a penicilina (Tabelas $1 \mathrm{e}$ 2), resultados estes equivalentes aos obtidos por Mishra ${ }^{9}$ e Diker, Akan e Harizoglu ${ }^{4}$. A resistência a estes antimicrobianos, poderia ser creditada à sua indicação primária contra agentes gram-positivos, e terapia alternativa contra microrganismos gram-negativos, incluindo a $M$ haemolytica e a $P$. multocida. ${ }^{10}$ Adicionalmente, das 17 fazendas estudadas, 16 usavam tetraciclina ou penicilina ou ambas, para o tratamento de toda e qualquer afecção que acometia os ovinos, inclusive doenças respiratórias. Por questões econômicas e/ou desconhecimento, a terapia nas fazendas era realizada sem o respaldo de testes de susceptibilidade in vitro, recaindo a escolha da droga, na maioria das vezes, na experiência de tratamentos anteriores, ou no 
Tabela 1 - Perfil de sensibilidade a antimicrobianos pelo teste de difusão de disco em 275 amostras de Mannheimia haemolytica, isoladas de cavidade nasal e de orofaringe de ovinos clinicamente sadios e de portadores de afecções respiratórias em 17 fazendas amostradas na região de Botucatu - SP

\begin{tabular}{lccccccc}
\hline \multirow{2}{*}{ Antimicrobiano } & \multicolumn{2}{c}{ Sensíveis } & \multicolumn{2}{c}{ Parcialmente } & \multicolumn{2}{c}{ Resistentes } & Total \\
\cline { 2 - 8 } & $\mathrm{n}$ & $\%$ & $\mathrm{n}$ & $\%$ & $\mathrm{~N}$ & $\%$ & $\mathrm{n}$ \\
\hline Enrofloxacina & 275 & 100 & 0 & 0 & 0 & 0 & 275 \\
Florfenicol & 275 & 100 & 0 & 0 & 0 & 0 & 275 \\
Ciprofloxacina & 274 & 99,64 & 1 & 0,36 & 0 & 0 & 275 \\
Sulfametazol + & 274 & 99,64 & 0 & 0 & 1 & 0,36 & 275 \\
Trimetoprim & 264 & 96 & 6 & 2,18 & 5 & 1,82 & 275 \\
Penicilina & 224 & 81,45 & 8 & 2,91 & 43 & 15,64 & 275 \\
Tetraciclina & & & & & & & \\
\hline
\end{tabular}

$\mathrm{n}=$ número de cepas testadas

Tabela 2 - Perfil de sensibilidade a antimicrobianos pelo teste de difusão de disco em 119 amostras de Pasteurella multocida, isoladas de cavidade nasal e de orofaringe de ovinos clinicamente sadios e de portadores de afecções respiratórias em 17 fazendas amostradas na região de Botucatu - SP

\begin{tabular}{lccccccc}
\hline \multirow{2}{*}{ Antimicrobiano } & \multicolumn{2}{c}{ Sensíveis } & \multicolumn{2}{c}{$\begin{array}{c}\text { Parcialmente } \\
\text { sensíveis }\end{array}$} & \multicolumn{2}{c}{ Resistentes } & Total \\
\cline { 2 - 8 } & $\mathrm{n}$ & $\%$ & $\mathrm{n}$ & $\%$ & $\mathrm{~N}$ & $\%$ & $\mathrm{n}$ \\
\hline Enrofloxacina & 119 & 100 & 0 & 0 & 0 & 0 & 119 \\
Florfenicol & 119 & 100 & 0 & 0 & 0 & 0 & 119 \\
Ciprofloxacian & 117 & 98,32 & 2 & 1,68 & 0 & 0 & 119 \\
Sulfametazol + & & & & & & & \\
Trimetoprim & 118 & 99,16 & 0 & 0 & 1 & 0,84 & 119 \\
Penicilina & 112 & 94,12 & 2 & 1,68 & 5 & 4,20 & 119 \\
Tetraciclina & 92 & 77,31 & 6 & 5,04 & 21 & 17,65 & 119 \\
\hline
\end{tabular}

$\mathrm{n}=$ número de cepas testadas

apelo comercial de determinados produtos.

Enrofloxacina, florfenicol, ciprofloxacina, sulfametoxazol/trimetoprim foram os antimicrobianos que apresentaram maior eficiência contra as cepas de $M$. baemolytica e P. multocida isoladas, enquanto que as maiores freqüências de resistência para esses agentes foram observadas frente à tetraciclina.

\section{Agradecimentos}

Os autores agradecem a Fundação de Amparo a Pesquisa do Estado de São Paulo - FAPESP. 


\title{
In vitro susceptibility to antimicrobials of Mannheimia haemolytica and Pasteurella multocida isolated from healthy ovine and with respiratory diseases
}

\begin{abstract}
Pasteurella multocida and Mannheimia haemolytica (P. haemolytica) are associated with ovine respiratory diseases. With the purpose of observe the susceptibility in vitro of these organisms against antimicrobials, were collected samples of nasopharingeal $(\mathrm{n}=180)$ and oropharingeal $(\mathrm{n}=82)$ from ovines healthy and with respiratory disease. Among the antimicrobials tested, the sensibility was greater for enrofloxacin $(100 \%)$ and florfenicol $(100 \%)$, for both bacteria. The greater resistance indices for $M$. baemolytica and $P$. multocida were observed with tetracyclin (15.64\% and $17.65 \%$ respectively) and penicillin $(1.82 \%$ and $4.2 \%)$.
\end{abstract}

\section{Referências}

1 CHANDRASEKARAN, S.; HIZAT, K.; SAAD, Z.; JAHARA, M. Y.; YEAP, P. C. Evaluation of combined Pasteurella vaccines in control of sheep pneumonia. British Veterinary Journal, v. 147, p. 437-443, 1991.

2 MARTIN, W. B. Respiratory infections of sheep. Comparative Immunology Microbiology Infectious Diseases, v. 19, p. 171-179, 1996.

3 GONÇALVES, R. C. Estudo clínico e citológico em bezerros clinicamente sadios e portadores de broncopneumonia moderada e grave - o lavado traqueobrônquico como complemento diagnóstico, SP. 1997. 144 f. Tese (Doutorado) - Faculdade de Medicina Veterinária e Zootecnia, Universidade Estadual Paulista, Botucatu, SP, 1997.

4 DIKER, K. S.; AKAN, M.; HARIZOGLU, R. Antimicrobial susceptibility of Pasteurella haemolytica and Pasteurella multocida isolated from pneumonic ovine lungs. Veterinary Record, v. 4, p. 597-598, 1994.

5 MACKIE, J. T.; BARTON, M.; HINDMARSH, M. Pasteurella haemolytica septicemia in sheep. Veterinary Record, v. 5, p. 245, 1995.

\author{
Key words: \\ Antimicrobials. \\ Mannheimia haemolytica. \\ Ovine. \\ Pasteurella multocida. \\ Respiratory diseases.
}

6 STÖBER, M. Aparelho Respiratório. In: DIRKSEN, G.; GRÜNDER, H. D.; STÖBER, M. Exame clínico dos bovinos. 3 ed. Rio de Janeiro: Guanabara Koogan, 1993. p. 139-165.

7 VIANA, L. Pesquisa de Mannheimia (Pasteurella) haemolytica e Pasteurella multocida em ovinos clinicamente sadios e portadores de afecções respiratórias na região de Botucatu-SP, SP. 2003. 76 f. Dissertação (Mestrado) - Faculdade de Medicina Veterinária e Zootecnia, Universidade Estadual Paulista, Botucatu, SP, 2003

8 BAUER, I.; KIRBY, I.; SHERRIS, I.; TUCK, M. Antibiotic susceptibilidad testing by a standardized single disk method. American Journal Clinical Pathology, v. 45, p. 1021-1026, 1966.

9 MISHRA, K. C. Study on microbial flora of the upper respiratory tracty of pneumonia-prone flocks of sheep end goats. Indian Journal Animal Science, v. 18, p. 27 33, 1988.

10 TAVARES, W. Manual de antibióticos e quimioterápicos infeciosos. 2. ed. São Paulo: Ateneu, 1996. 792 p. 